\title{
SEX IN GRAPHS ${ }^{1}$
}

\section{OYSTEIN ORE}

1. The problem. The present study in graph theory is inspired by a problem arising in the field of biology. Let $V$ be a population of some sort consisting of individual organisms capable of sexual reproduction. The interrelations of the members in $V$ in regard to descendence from each other may then be described by means of a binary progeny relation $\pi$ such that

$$
a \pi b, \quad a, b \in V
$$

whenever $b$ is a progeny (child, off-spring) of the individual $a$. To $\pi$ corresponds a directed graph $G=G(\pi)$, the reproduction graph for $V$. It shall have single edges and no loops; there is an edge $(a, b)$ in $G$ if and only if $b$ is a progeny of $a$. When there exists a directed path $P(c, d)$ between two vertices we write $c>d$ and call $d$ a descendant of $c$ and $c$ a predecessor of $d$. The corresponding descendence graph is seen to be a partial order.

According to our assumptions the vertex set $V$ must fall into two disjoint sexual groups, males $m$ and females $f$ such that

$$
V=M+F, \quad m \in M, f \in F .
$$

By the mating of two individuals of opposite sex one or more offspring will result. There are two immediate properties of this reproduction process:

1. Each individual has exactly two parents.

2. No individual is its own descendant.

In graph terminology this means:

1. The number of incoming edges at each vertex $a \in V$ is

$$
\rho^{*}(a)=2 .
$$

(In general $\rho(a)$ and $\rho^{*}(a)$ are the local degrees at $a$, that is, the number of outgoing and incoming edges.)

2. The graph is acyclic, that is, there are no directed cyclic paths.

We shall here examine the graph implications of these properties and pose the following question: Suppose that in a directed graph the two preceding conditions are satisfied. When is it possible to assign a sex to the vertices of $G$, that is, when does there exist a disjoint

Received by the editors October 8, 1959.

1 This paper was prepared with the assistance of a National Science Foundation research grant. 
decomposition (1.1) of the vertex set such that at each vertex there is one edge coming from each set?

2. Alternating paths. Before we analyze the preceding problem we shall discuss certain concepts concerning directed graphs which are of importance also for other questions. A sequence of edges in a directed graph $G$

$$
P=\left(a_{0}, a_{1}\right)\left(a_{2}, a_{1}\right)\left(a_{2}, a_{3}\right)\left(a_{4}, a_{3}\right) \cdots
$$

shall be called an alternating sequence; it is an alternating path when no edge appears more than once. One can also define an alternating sequence as a sequence

$$
P=\left(a_{0}, a_{1}\right)\left(a_{1}, a_{2}\right)\left(a_{2}, a_{3}\right) \cdots\left(a_{n-1}, a_{n}\right)
$$

in which the edges $E_{i}=\left(a_{i}, a_{i+1}\right)$ alternately belong to $G$ and its converse $G^{*}$, that is, the graph with reversed directions on the edges. Such sequences shall be classified according to the character of the initial edge $E_{0}$. It is an $\alpha$-sequence when $E_{0}$ is an $\alpha$-edge, that is, belongs to $G$. It is an $\alpha^{*}$-sequence when $E_{0}$ is an $\alpha^{*}$-edge, that is, belongs to $G^{*}$. Often one wishes to take into account also the character $\beta=\alpha$ or $\beta=\alpha^{*}$ of the last edge $E_{n-1}$. For instance, $P$ is an $\left(\alpha, \alpha^{*}\right)$ sequence when $E_{0}$ is in $G$ and $E_{n-1}$ in $G^{*}$. This yields four types of sequences characterized by the symbols

$$
(\beta, \gamma)=\left(\alpha, \alpha^{*}\right),\left(\alpha^{*}, \alpha\right),(\alpha, \alpha),\left(\alpha^{*}, \alpha^{*}\right) .
$$

The two first types of sequences have even lengths while the others have odd lengths. An alternating path or sequence is cyclic when $a_{0}=a_{n}$. We also extend the concept of alternating sequences to oneand two-way infinite sequences. When $P$ in $(2.2)$ is a $(\beta, \gamma)$-sequence we say that $a_{n}$ is $(\beta, \gamma)$-accessible from $a_{0}$ while $a_{0}$ is $\left(\gamma^{*}, \beta^{*}\right)$-accessible from $a_{n}$.

THEOREM 2.1. When the vertex $b$ is $(\beta, \gamma)$-accessible from the vertex $a$ and $c$ a vertex $\left(\gamma^{*}, \delta\right)$-accessible from $b$ then $c$ is $(\beta, \delta)$-accessible from $a$.

Proof. If the two connecting sequences $P(a, b)$ and $Q(b, c)$ have no edges in common they can be combined into a single $(\beta, \delta)$-sequence

$$
P(a, b)+Q(b, c) \text {. }
$$

When they have common edges let $E=(e, f)$ be the first such edge in $P$. One of the sequences

$$
P(a, e, f)+Q(f, c), \quad P(a, e)+Q(e, c)
$$


is then a $(\beta, \delta)$-sequence depending on whether $E$ appears in the same or opposite directions in $P$ and $Q$.

3. Accessible sets. The set of all $(\beta, \gamma)$-accessible vertices from a vertex $a$ we denote by

$$
V(a ; \beta, \gamma) .
$$

This definition can be extended to arbitrary sets $A$ by putting

$$
V(A ; \beta, \gamma)=\sum_{a} V(a ; \beta, \gamma),
$$

From the definition of these accessible sets one verifies the relations

$$
\begin{aligned}
V\left(G[A] ; \alpha^{*}, \alpha\right) & =V(A ; \alpha, \alpha), \\
V\left(A ; \alpha, \alpha^{*}\right) & =A+V\left(G[A] ; \alpha^{*}, \alpha^{*}\right)
\end{aligned}
$$

where $G[A]$ is the $G$-set of $A$, that is, the endpoints of edges in $G$ having their initial vertex in $A$. From (3.3) one sees that there are essentially only two types of such accessible sets, namely those of the forms

$$
V(A)=V\left(A ; \alpha, \alpha^{*}\right), \quad V^{*}(A)=V\left(A ; \alpha^{*}, \alpha\right) .
$$

These sets can also be characterized as follows:

Theorem 3.1. A set $A$ is an $\left(\alpha, \alpha^{*}\right)$-accessible set if and only if all incoming edges to $G[A]$ have their initial vertex in $A$, that is,

$$
G^{*}[G[A]]=A .
$$

Proof. It is evident that this property insures

$$
A=V\left(A ; \alpha, \alpha^{*}\right) \text {. }
$$

On the other hand, if there should be some edge

$$
F=\left(a^{\prime}, a^{\prime \prime}\right), \quad a^{\prime \prime} \notin A, a^{\prime} \in G[A]
$$

one could construct an $\left(\alpha, \alpha^{*}\right)$-path $E+F$ to $a^{\prime \prime}$, hence $A$ would not have the form (3.6).

We denote by

$$
\rho(A)=\sum_{a} \rho(a), \quad a \in A,
$$

the sum of all local degrees for the vertices in the set $A$, hence the number of edges in $G$ issuing from the vertices in $A$. From Theorem 3.1 follows a special case: 
Theorem 3.2. A finite set $A$ with finite local degrees $\rho(a)$ is an $\left(\alpha, \alpha^{*}\right)$-accessible set if and only if

$$
\rho(A)=\rho^{*}(G[A]) .
$$

Next we mention:

Theorem 3.3. The two families of accessible sets (3.4) form two complete fields of sets.

Proof. The definition (3.2) shows that any sum of sets $V(A)$ is of the same type. When

$$
d \in D=\Lambda V_{i}
$$

is a vertex belonging to an intersection of accessible sets then also $V(d)$ must belong to $D$. Finally it must be shown that a difference set

$$
V_{1}=V(A)-V(B), \quad V(A) \supset V(B)
$$

is an accessible set. For any edge

$$
E=\left(a, a^{\prime}\right),
$$$$
a \in V_{1}
$$

one must have

$$
a^{\prime} \in G[V(A)]
$$

and so for any other edge $F=\left(a^{\prime \prime}, a^{\prime}\right)$ to $a^{\prime}$ also $a^{\prime \prime} \in V(A)$. But $a^{\prime \prime} \in V(B)$ is not possible since the path $F+E$ would lead from $a^{\prime \prime}$ to the vertex $a$ not in $V(B)$.

4. Accessibly equivalent vertices. Two vertices $a$ and $b$ are $\alpha$ accessibly equivalent when any vertex $v$ which is $(\alpha, \beta)$-accessible from $a\left(\beta=\alpha\right.$ or $\left.\beta=\alpha^{*}\right)$ is also $(\alpha, \beta)$-accessible from $b$ and vice versa. We consider $a$ to be $\alpha$-accessibly equivalent to itself. We shall show :

TheOREM 4.1. The accessible set $V(a)$ consists of all vertices $\alpha$-acessibly equivalent to $a$.

Proof. First we observe that when $b \in V(a)$ there exists an $\left(\alpha, \alpha^{*}\right)$ path $P(a, b)$ with a reverse $\left(\alpha, \alpha^{*}\right)$-path $P(b, a)$, hence $a$ and $b$ must be $\alpha$-accessibly equivalent according to Theorem 2.1. Secondly, suppose that $a$ and $b$ are $\alpha$-accessibly equivalent. Since $a$ must be $\alpha$-accessible from $b$ there is an $\alpha$-path $P(b, a)$. Again by Theorem 2.1 we need only examine the case where $P$ is an $(\alpha, \alpha)$-path. Let $c$ be the last vertex in $P(b, a)$ before $a$ such that $c$ is $\left(\alpha, \alpha^{*}\right)$-accessible from $b$. By assumption $c$ is $\left(\alpha, \alpha^{*}\right)$-accessible from $a$ in a path $Q(a, c)$. But then 


$$
Q(a, c)+P(c, b)
$$

produces an $\left(\alpha, \alpha^{*}\right)$-sequence from $a$ to $b$ so that $b \in V(a)$.

Similarly one finds that the set $V^{*}(a)$ consists of all vertices which are $\alpha^{*}$-accessibly equivalent to $a$. One can also say that a vertex $b$ is $\left(\alpha, \alpha^{*}\right)$-accessibly equivalent to $a$ when any vertex accessible from $a$ in an $(\alpha, \beta)$-path is accessible from $b$ in an $\left(\alpha^{*}, \beta\right)$-path and conversely. One verifies that the set of all such vertices form the set

$$
V\left(G[a] ; \alpha^{*}, \alpha\right)=G[V(a)] .
$$

5. The alternating composition graph. The alternating composition graph $G\left(\alpha, \alpha^{*}\right)$ of a directed graph $G$ is defined as the graph product

$$
G\left(\alpha, \alpha^{*}\right)=G \cdot G^{*}
$$

of $G$ and its converse $G^{*}$. This means that $G\left(\alpha, \alpha^{*}\right)$ has the same vertex set $V$ as $G$ and two vertices $a$ and $c$ are connected by an edge $E_{\alpha}=(a, c)$ in $G\left(\alpha, \alpha^{*}\right)$ if and only if $a$ and $c$ are connected by an alternating $\left(\alpha, \alpha^{*}\right)$-path of length 2 in $G$, that is, there exist edges

$$
E_{1}=(a, b), \quad E_{2}=(b, c)
$$

in $G$ and $G^{*}$ respectively. One sees that the graph $G\left(\alpha, \alpha^{*}\right)$ can be considered to be undirected. The preceding discussion shows that its connected components are the accessible sets $V(a)$. There may be multiple edges. Denote by $M(G)$ the graph matrix of $G$, that is,

$$
M(G)=\left(a_{i j}\right)
$$

where $a_{i j}=1$ or $a_{i j}=0$ depending on whether or not there is an edge $(i, j)$ in $G$. Then the matrix of the alternating composition graph is the product

$$
M\left(G \cdot G^{*}\right)=M(G) \cdot M\left(G^{*}\right)
$$

with the multiplicities of the edges taken into account. One can also define the converse alternating composition graph

$$
G\left(\alpha^{*}, \alpha\right)=G^{*} \cdot G .
$$

Its connected components are the sets $V^{*}(a)$.

6. The dichotomy problem. We shall now return to the biological graph problem introduced in $\S 1$. We prefer to consider it first in a somewhat more general form by dropping the condition that $G$ shall be acyclic. Furthermore we shall assume instead of (1.2) that only the weaker condition 


$$
\rho^{*}(a) \leqq 2
$$

holds. This can be expressed that each progeny has at most two parent vertices.

In such a graph we assign the sexual character $m$ to some arbitrary vertex $a_{0}$. If $a_{0}$ has a progeny $a_{1}$ then $a_{1}$ may also be the progeny of some other parent $a_{2}$ In this case $a_{2}$ must be assigned the character $f$. From $a_{2}$ one proceeds in the same manner: When $a_{2}$ has some progeny $a_{3}$ and $a_{3}$ some other parent $a_{4}$ then $a_{4}$ must have the character $m$. By continuing this process a character is assigned to all vertices $a_{2 n}$ accessible from $a_{0}$ in $\left(\alpha, \alpha^{*}\right)$-paths, that is, using the terminology of $\$ 3$, to the vertices in the set

$$
V\left(a_{0} ; \alpha, \alpha^{*}\right) .
$$

However, in order that the characters $m$ and $f$ thus obtained be unique all paths to $a_{2 n}$ must assign the same character. One sees readily that for this to be the case it is necessary and sufficient that the lengths $l$ of all cyclic $\left(\alpha, \alpha^{*}\right)$-sequences be divisible by 4 . When this condition is satisfied characters with the desired properties can be assigned in each set (6.2).

For a general directed graph let us use the terminology that the vertex $a$ is a parent of $b$ when there is an edge $(a, b)$ from $a$ to $b$. We shall also say that $G$ has a sex dichotomy when there exists a disjoint decomposition (1.1) such that each individual with at least two parents has exactly two of them, one from each set $M$ and $F$. The preceding analysis then yields:

TheOREm 6.1. A necessary and sufficient condition that a directed graph have a sex dichotomy is that the length of all cyclic alternating $\left(\alpha, \alpha^{*}\right)$-sequences be divisible by 4 .

It should be noted that in the formulation of this result the condition (6.1) has been omitted. This may be done since it is actually a consequence of the condition on the sequences. If namely there should be as many as 3 incoming edges to any vertex then one readily verifies that there is a cyclic $\left(\alpha, \alpha^{*}\right)$-sequence in $G$ of length 6 .

Theorem 6.1 can be reformulated in terms of the alternating composition graph $G \cdot G^{*}$ introduced in (5.1). An $\left(\alpha, \alpha^{*}\right)$-sequence in $G$ corresponds to a unique edge sequence in $G \cdot G^{*}$. When the cyclic $\left(\alpha, \alpha^{*}\right)$-sequences in $G$ have lengths which are divisible by 4 the cyclic paths in $G \cdot G^{*}$ have even lengths, hence $G \cdot G^{*}$ is a bipartite graph and vice versa. Furthermore, the sets (6.2) are the connected components of $G \cdot G^{*}$. In each of them one can assign the character $m$ 
or $f$ to a given vertex $a_{0}$ and the characters of the remaining vertices are uniquely determined. Thus Theorem 6.1 can be stated:

Theorem 6.2. A necessary and sufficient condition that a directed graph $G$ have a sex dichotomy is that the alternating composition graph $G \cdot G^{*}$ be bipartite. The number of ways in which the characters can be assigned is $2^{k}$ where $k$ is the number of connected components of $G \cdot G^{*}$.

7. The reproduction graphs. Let us return to the reproduction graph. One can construct such a graph successively by letting pairs of vertices $a$ and $b$ give rise to new edges $(a, c)$ and $(b, c)$ where the vertex $c$ cannot coincide with any of the predecessors of $a$ or $b$. If in this acyclic graph every individual has two parents the graph must be infinite. In genetic experiments or descriptions of them one usually starts from some given limited population or generating set $B$ from which the subsequent generations are produced. The predecessors to these individuals are unknown and one puts $\rho^{*}(b)=0$ in $B$. One might also begin with some generating set in which the family relations are only incompletely known. Thus one is led to consider the condition (6.1) as being satisfied for the general reproduction graphs. By this definition one concludes from Theorem 6.2:

Theorem 7.1. A directed graph $G$ is a reproduction graph if and only if it is acyclic and its alternating composition graph $G \cdot G^{*}$ is bipartite.

In a reproduction graph one can introduce the usual concepts of family relationships: Father, mother, brother, sister, half-brother, half-sister, uncle, aunt, cousin, etc. Most of the common taboos of human society can be represented as simple restrictions on the reproduction graph. For instance, mating with a descendant is not permitted. In graph terms this means that when there exists a directed path $P\left(a_{0}, a_{n}\right)$ there can exist no edge $\left(a_{0}, a_{n}\right)$, in other words, the reproduction graph must be a basis graph. The reader may verify the graph formulation of various other taboos, for instance, against marriage between siblings or half-siblings. Other conditions severally restrict the graph, for instance, a rule of absolute monogamy for one of the sexes or both.

For a reproduction graph $G$ the composition graph $G \cdot G^{*}$ could also suitably have been called the mating graph. The multiplicity $\rho(a, b)$ of edges connecting two vertices $a$ and $b$ in $G \cdot G^{*}$ indicates the number of off-spring having been produced by the mating of the two corresponding individuals. 